\title{
Asymmetric Dimethylarginine
}

National Cancer Institute

\section{Source}

National Cancer Institute. Asymmetric Dimethylarginine. NCI Thesaurus. Code C124264.

A methylated derivative of $\mathrm{L}$-arginine that is a competitive inhibitor of nitric oxide synthase (NOS). Asymmetric dimethylarg inine (ADMA) is formed when Sadenosylmethionine protein $\mathrm{N}$-methyltransferases transfer methyl groups from $\mathrm{S}$ adenosylmethionine to arginine. Free ADMA in plasma competes with the L-arginine for binding to the heme in NOS and inhibits nitric oxide (NO) synthesis. Decreased synthesis of NO inhibits vasodilation and leads to endothelial dysfunction. Elevated plasma levels of ADMA are seen in certain types of cancer, cardiovascular disease, hypertension, hyperlipidemia, type 2 diabetes mellitus, and increased oxidative stress. 